This item was submitted to Loughborough's Research Repository by the author.

Items in Figshare are protected by copyright, with all rights reserved, unless otherwise indicated.

\title{
Planar electromagnetic bandgap structures based on polar curves and mapping functions
}

\section{PLEASE CITE THE PUBLISHED VERSION}

PUBLISHER

(c) IEEE

VERSION

VoR (Version of Record)

\section{LICENCE}

CC BY-NC-ND 4.0

\section{REPOSITORY RECORD}

Mulenga, Charity B., and James A. Flint. 2019. "Planar Electromagnetic Bandgap Structures Based on Polar Curves and Mapping Functions". figshare. https://hdl.handle.net/2134/6121. 
This item was submitted to Loughborough's Institutional Repository (https://dspace.lboro.ac.uk/) by the author and is made available under the following Creative Commons Licence conditions.

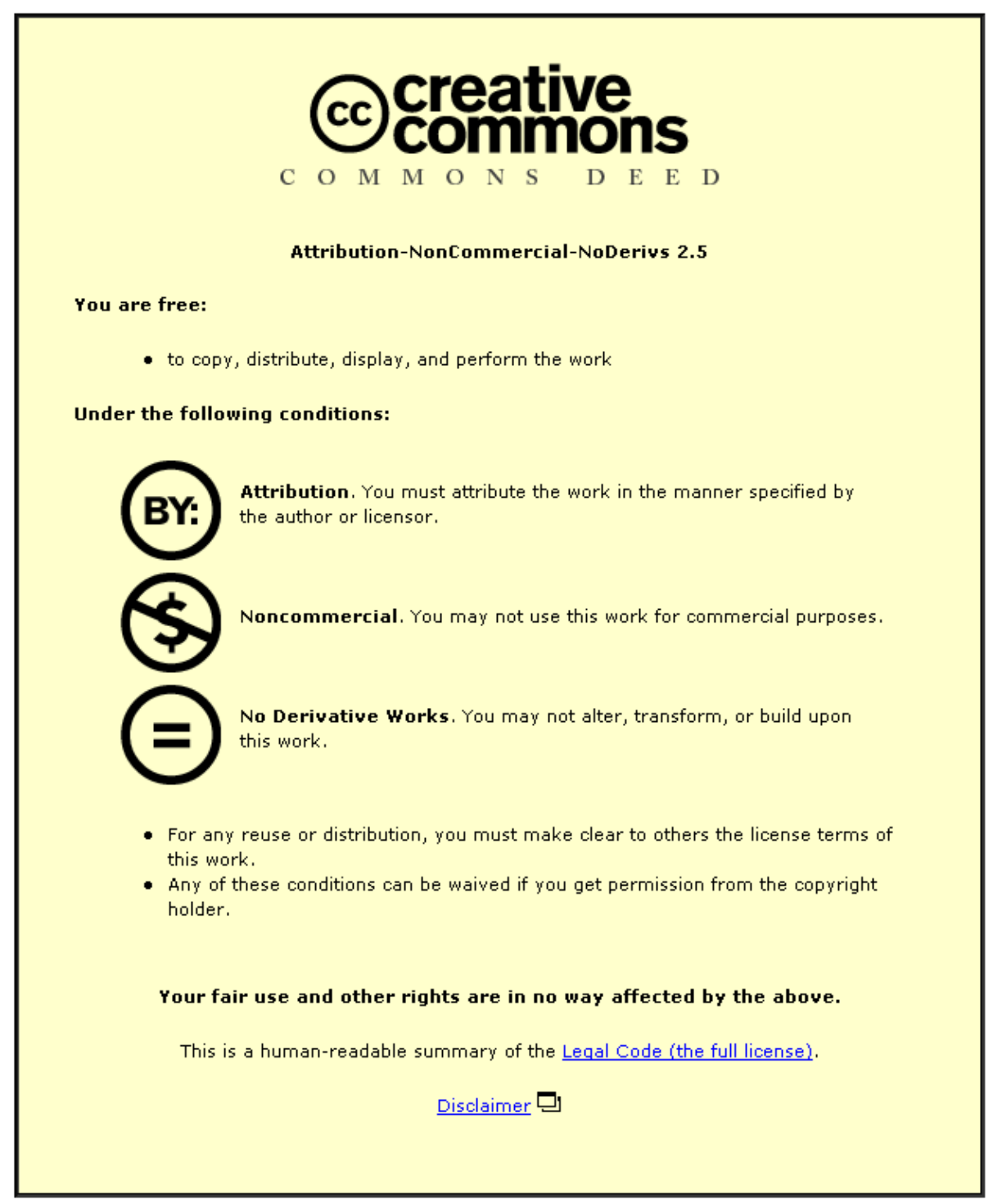

For the full text of this licence, please go to: http://creativecommons.org/licenses/by-nc-nd/2.5/ 


\title{
Planar Electromagnetic Bandgap Structures Based on Polar Curves and Mapping Functions
}

\author{
Charity B. Mulenga and James A. Flint, Senior Member, IEEE
}

\begin{abstract}
A type of electromagnetic bandgap structure is described that is easily parameterized and can produce a range of square and spiral geometries. Individual electromagnetic bandgap (EBG) geometries are defined on a cell-by-cell basis in terms of their convolution factor $k$, which defines the extent to which the elements are interleaved and controls the coupling slot length between adjacent elements. Polar equations are used to define the slot locus which also incorporate a transformation which ensures the slot extends into the corners of the square unit cell and hence extends the maximum slot length achievable. The electromagnetic properties of the so-called polar $E B G$ are evaluated by means of numerical simulation and measurements and dispersion diagrams are presented. Finally, the performance is compared with other similar miniaturized EBG cell geometries. It is shown that the polar EBG has better angular stability than the equivalent square patch design and is comparable in terms of performance to other low frequency EBG elements. At the same time it retains the ability to fine tune the response by adjusting $k$.
\end{abstract}

Index Terms-Electromagnetic bandgap (EBG), mapping functions, metamaterials, polar curves.

\section{INTRODUCTION}

O VER the past decade periodic structures such as electromagnetic bandgap (EBG) materials have attracted extensive research interest in the microwave and millimeter wave domains. The major characteristic of EBG surfaces is the existence of one or more bands whereby propagating surface waves are effectively suppressed. In addition, many of these structures also have the property that they reflect normally-incident plane waves with a reflection coefficient of $\Gamma=+1$ within certain frequency bands. In this case the surface behaves as a wall of magnetic symmetry and hence is often termed an artificial magnetic conductor (AMC) [1], [2]. There are numerous applications of these periodic metamaterials in the field of antenna design [3]-[9]. Specific uses for these materials include producing improved ground planes for individual antennas. [10], and reducing mutual coupling for patch antennas or arrays [7], [11]-[14]. Additionally, EBG structures have been applied to coplanar waveguides (CPW) to reduce leaky waves. [15], [16] and to realize directive base station antennas [17]. Other applications include the mitigation of switching noise in high-speed

\footnotetext{
Manuscript received May 06, 2009; revised July 16, 2009. First published December 31, 2009; current version published March 03, 2010. This work was supported in part by Metamorphose NoE and in part by Loughborough University.

The authors are with the Department of Electrical and Electronic Engineering, Loughborough University, Loughborough LE11 3TU, U.K. (e-mail: james.flint@ieee.org).

Color versions of one or more of the figures in this paper are available online at http://ieeexplore.iee.org.

Digital Object Identifier 10.1109/TAP.2009.2039319
}

circuits [18]-[20] and improvement of signal isolation in RF mixed signal systems [20].

The properties of the EBG only exist within a specific band and thus careful design is necessary in order to select the resonant frequency $\left(f_{c}\right)$ and the bandwidth (BW) of a particular array geometry. For a given array the center frequency is given by [21]

$$
f_{c}=\frac{1}{2 \pi \sqrt{L C}}
$$

where $L$ and $C$ are the per unit area inductance and capacitance respectively. In the simplest element geometry, which consists of an array of square patches shorted to ground at their centers, the capacitance is mainly due to fringing fields between elements and the inductance is mainly due to the current loop length between adjacent vias [1]. The bandwidth is also an important consideration and is given by [21]

$$
\mathrm{BW}=\frac{1}{\eta} \sqrt{\frac{L}{C}}
$$

where $\eta$ is the free space impedance. In low frequency applications, it is often the case that the array becomes physically very large for a given number of cell periods and thus it is necessary to apply special methods and geometries in order to increase $L$ and $C$. The most desirable approach would be to increase the inductance as it can be seen in (2). This leads to a bandwidth improvement (e.g., by introducing meandering vias [22], [23], slanting vias [24], or high permeability materials [25]). However there are disadvantages to this approach such as introducing more difficult manufacturing processes or increasing losses. Another approach which can be taken in addition to increasing inductance, is increasing the capacitance between adjacent elements and that is the focus of the current paper.

Capacitance increase can be achieved by using high permittivity substrates [23], interleaving and convoluting elements [26], introducing inter-digitated edges between adjacent patches [6], [27] and by reducing the gap width between adjacent elements. It is also possible to produce thick conducting elements at the surface which exhibit both fringing and parallel-plate type capacitance.

Frequency-reducing geometries which have been proposed often make use of fractal space filling methods such as Hilbert and Peano curves. As the iteration order of the curve increases, the footprint is preserved while the length of the curve increases. The Hilbert curve geometry has been applied successfully to produce AMC structures with low resonant frequencies. [2], [28]. One issue with these element types is the necessity to select an integer order number which means that fine tuning of the 
resonant frequency has to be achieved by adjusting other parameters such as the array periodicity, substrate thickness and gap widths. It is an objective of the current paper to demonstrate an array element geometry which can be tuned without changing the substrate thickness or gap width, which are often inconvenient to change. In contrast, it is typically simple to modify the slot geometry on a substrate via photolithographic-etching or routing methods. If the array periodicity can be fixed between elements it also allows the frequency of different regions to be adjusted independently, potentially allowing geometrical perturbations in the surface to be corrected for.

This paper is organized as follows. In Section II, the concept of using polar curves and mapping functions in the design of low frequency EBG structures is introduced along with its resultant bandgap and AMC features. Section III discusses a method by which the surface can be tuned. The angular stability of polar-EBG structures is discussed in Section IV. Section V presents comparisons of the proposed geometry to well-known low frequency EBG structures, followed by conclusions in Section VI.

\section{Geometry and Performance of Polar EBGs}

\section{A. Polar EBG Geometry}

A diagram showing the upper surface of the proposed spirallike polar-EBG structure is shown in Fig. 1. The area in the center has been darkened in order to indicate the unit cell with the darkest areas representing the metallic areas in the array and the lighter parts representing the slots cut into the upper conductor. Below the upper surface is a layer of dielectric and finally a layer of conducting material below this to form a continuous ground plane. The ground plane is connected to the center of the metal patches in the array by a set of thin via pins which are labeled in Fig. 1.

If it is desired to increase the surface capacitance per unit area, then it is possible to decrease the width of the slot, but arguably a better way and an approach more conducive to easy manufacture is to increase the slot length. A straightforward method of defining the slot geometry is to make use of a polar equation which defines the locus of the slot (shown dotted in Fig. 1).

We define a non-integer factor, $k$, termed the convolution factor, which controls the extent to which the spiral geometry is modified. Higher convolution factors yields a geometry which tends towards the standard "mushroom" patch surface analyzed by Sievenpiper [1], lower numbers achieve spiral geometries of varying levels of convolution. The polar equation is shown in (3)

$$
F(\theta) \approx \frac{T}{\pi}\left\{w(\theta) \cdot \sin ^{-1}[\cos (k \theta)]\right\}
$$

where $F(\theta)$ describes the locus of the spiral slot shown dotted in Fig. 1 and other geometrical dimensions are as labeled. Equa-

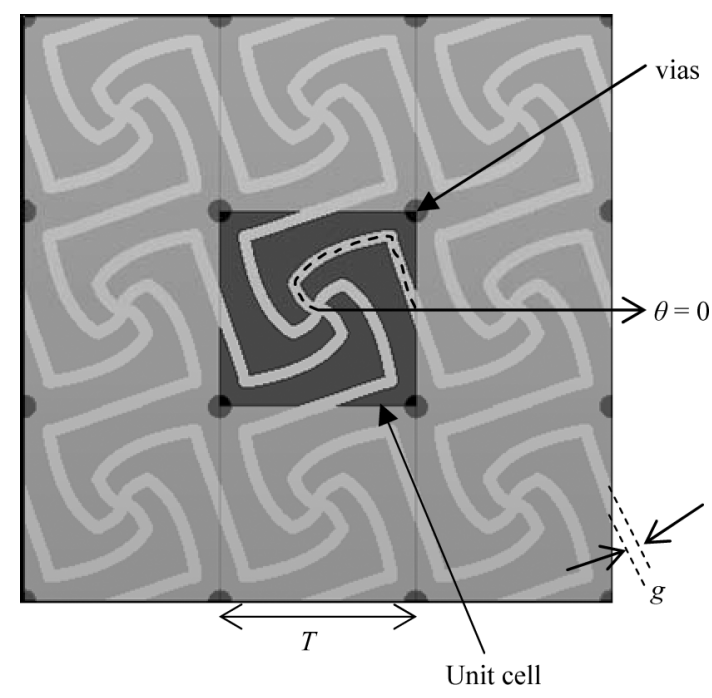

Fig. 1. Top view of the polar EBG structure (dark gray-copper). The locus of the slot (shown dotted in the unit cell) is defined by a polar equation.

tion (3) is derived by assuming that the center point of each individual slot within the unit cell of the original patch remains fixed, as do the extremities. This simple equation ensures that more convoluted slots (with lower $k$ values) will always remain equidistant as they cross the line of the original slot locus. The other slots in the unit cell are then determined by rotational symmetry. $w(\theta)$ is a mapping function which further modifies the polar function in order to better utilize the surface area available in the unit cell by extending the spiral-slot length. For $w(\theta)=1, F(\theta)$ generates the plain circular spiral-slots shown on the left hand side of Fig. 2. For a square unit cell, $w(\theta)$ can be chosen as the periodic function, as shown in (4) at the bottom of the page, where $n=0,1,2, \ldots$. The function, shown plotted in Fig. 3, produces warped spiral-slot patterns on the right hand side of Fig. 2. Note that these patterns are no longer idealized spirals but benefit from longer slot lengths due to their longer path within the unit cell.

The geometrical effects of changing the convolution factor and applying warping are clearly evident in Fig. 2. It can be seen that a variety of spiral and square-type EBG materials can generated by this technique which have varying properties, dependent on the factor $k$. The effect of varying $k$ on the slot length is shown in Fig. 4. The slot lengths shown are normalized to the square unit cell length, (i.e., $k \rightarrow \infty$ ), case. In theory it is possible to use extremely small values of $k$ with their corresponding long slots, however practical constraints due to the finite width of the slot, $g$, eventually prevent further reduction of $k$.

\section{B. Computer Simulation}

Periodic structures of the type described can be thought of as having two distinct properties- the AMC properties where the

$$
w(\theta)= \begin{cases}\frac{1}{\cos (\theta)} & \text { for } n \pi \leq \theta \leq(n+0.25) \pi,(n+0.5) \pi \leq \theta \leq(n+0.75) \pi \\ \frac{1}{\sin (\theta)} & \text { for }(n+0.25) \pi<\theta<(n+0.5) \pi,(n+0.75) \pi<\theta<(n+1) \pi\end{cases}
$$




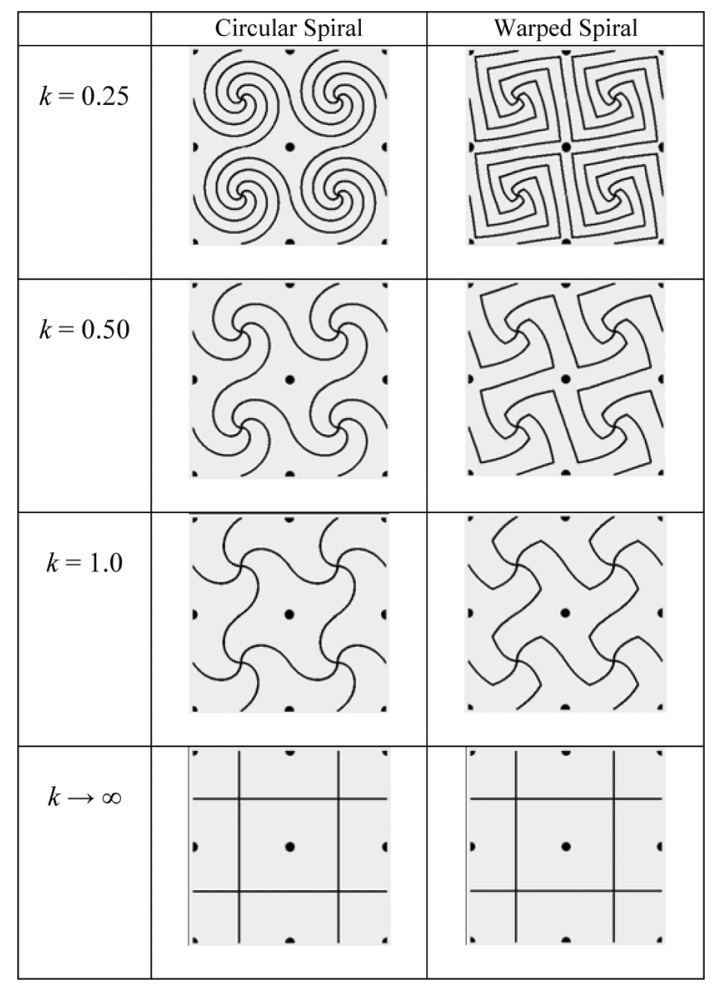

Fig. 2. Schematic of $2 \times 2$ Polar-EBG array showing the effects of changing the curve order on the length of the spiral. The lines indicate the slots cut into the upper conductive layer.

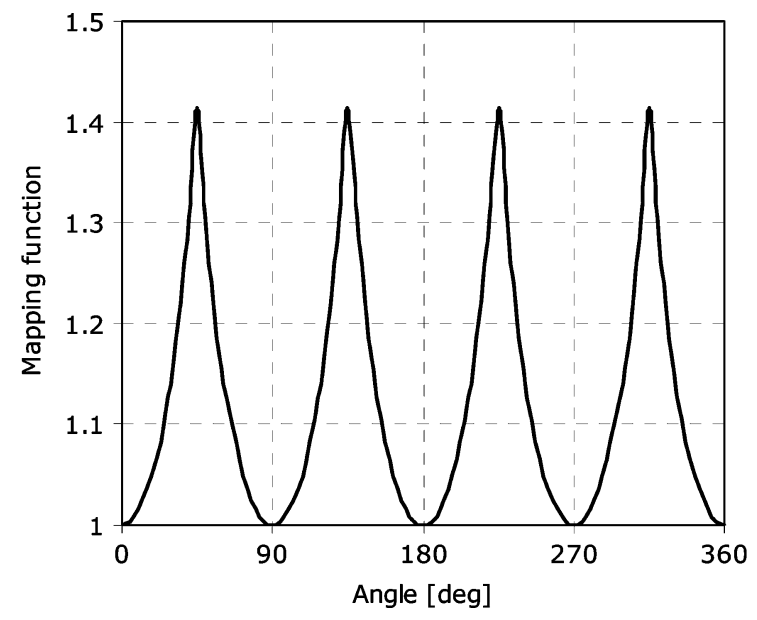

Fig. 3. Mapping function that provides a warping transformation.

reflected wave at the surface is considered to be more in phase with the incident wave than out of phase and the EBG property in which surface waves are suppressed. In this section the two properties are characterized for the spiral-slot based polar-EBG structure.

To verify the AMC property a polar-EBG and a conventional square patch EBG structure having equal periodicity, substrate material, and slot width were compared. The EBG structures were analyzed using the TLM method and a periodic boundary condition [29]. The cells were modeled on a dielectric slab 3.18 $\mathrm{mm}$ thick with relative permittivity of 2.95 . The length of the conventional square patch $(W)$ used was $7 \mathrm{~mm}$. In both cases

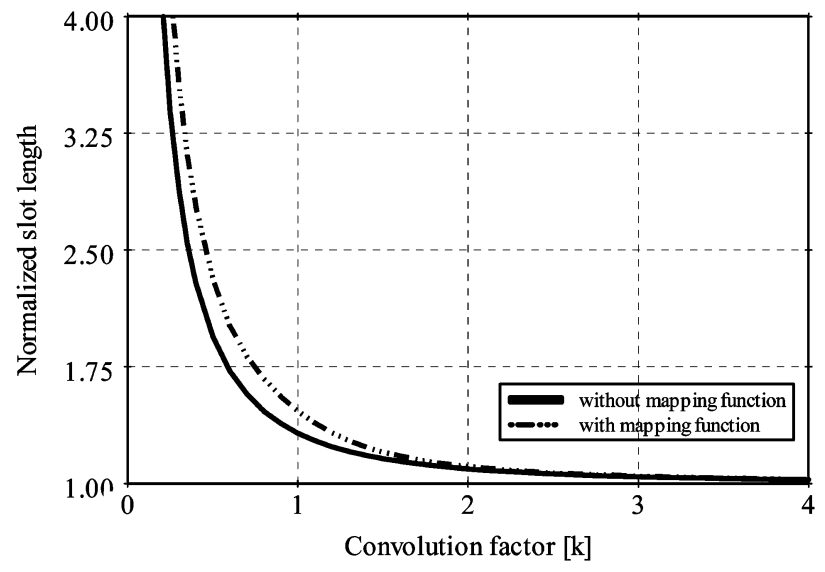

Fig. 4. Variation of slot length with convolution factor, $k$.

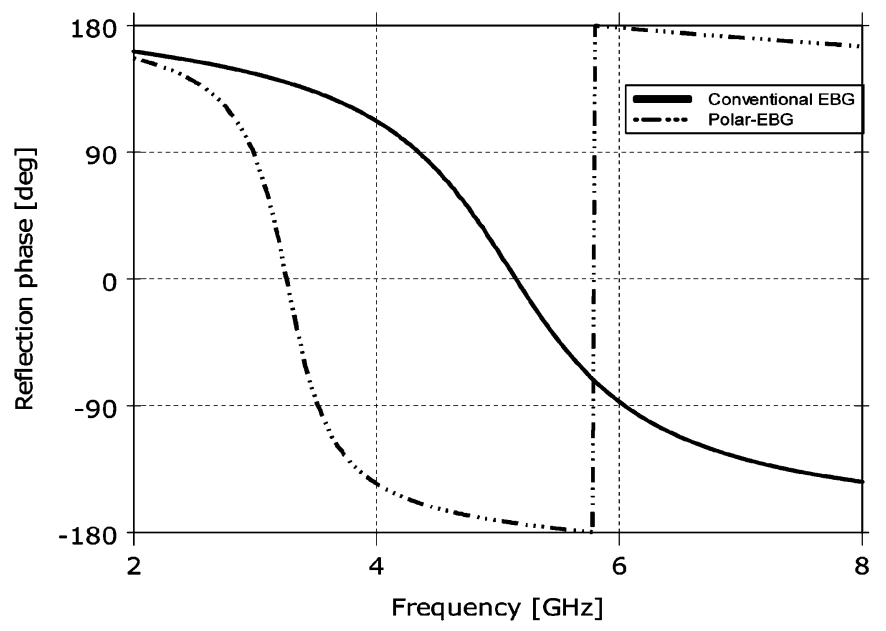

Fig. 5. Reflection phase comparison between the conventional EBG and Polar-EBG with equal periodicity $\mathrm{a}=7.5 \mathrm{~mm}, \mathrm{k}=0.5$ and $\mathrm{g}=0.5 \mathrm{~mm}$.

the array periodicity was $T=7.5 \mathrm{~mm}$ and the slot width, $g=$ $0.5 \mathrm{~mm}$.

The polar-EBG structure was designed using a convolution factor $k=0.5$ which gave the shape seen already in Fig. 2. The simulated reflection phase results are shown in Fig. 5. The frequency band where the EBG surface had a reflection phase in the range $\pm 90^{\circ}$ was taken to be the useable bandwidth. The reflection phase variation with frequency indicates a similar behavior in both cases, however the EBG has a 30\% frequency reduction compared to the conventional EBG. An expected reduction is also seen in the bandwidth in case of the polar-EBG. The lower resonant frequency is primarily due to first resonance of the structure and can be related directly to the spiral-slot length. Additionally, Fig. 5 demonstrates the viability of the polar-EBG as an artificial magnetic conductor.

The stop-band and pass-band frequencies of an EBG structure can be more fully expressed by using dispersion diagrams. Dispersion analysis has the main advantage of estimating the stop-bands of EBG structures without considering the entire structure. An infinite structure is simulated by imposing periodic boundary conditions with appropriate phase shifts onto the unit cell in a suitable eigenmode solver. The wave propagation in the structure can be represented by certain vectors in the unit cell 


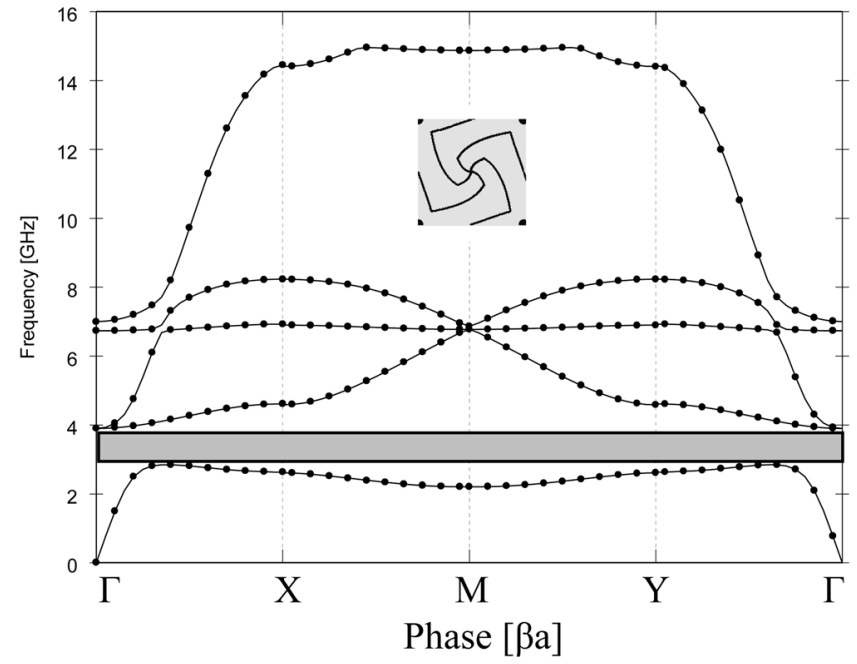

(a)

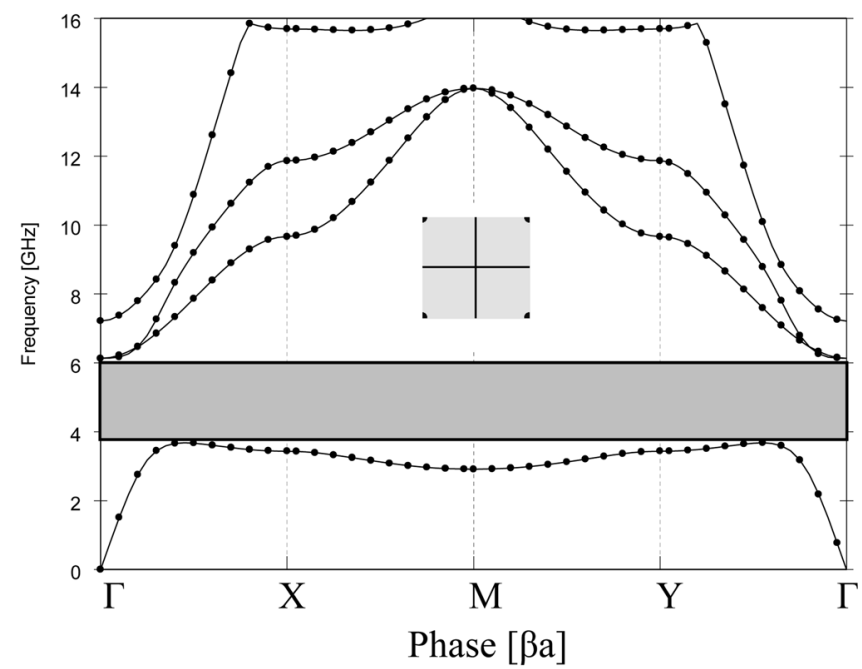

(b)

Fig. 6. Dispersion diagram for the Polar-EBG structures (a) Polar-EBG k = 0.5 (b) Conventional EBG.

that constitute a boundary region of propagation, often referred to as the irreducible Brillouin zone. Deriving the propagating modes in this zone suffices to cover all the possible directions of propagation within the unit cell. The Polar-EBG structure has its irreducible Brillouin zone as the entire unit cell given that it has rotational symmetry and not reflective symmetry. Fig. 6 shows the full $\Gamma-X-M-Y-\Gamma$ dispersion diagram of the Polar-EBG structure and a conventional EBG structure. The dispersion diagrams have been calculated using an eigenmode solver based on the Finite Integration Method [15]. The Polar-EBG exhibits a bandgap from $2.8-3.9 \mathrm{GHz}$ while the conventional EBG shows a bandgap from 4-6.2 GHz. The stopbands predicted by the dispersion diagrams show very good agreement with those predicted through reflection phase analysis. The suppression of surface waves plays a useful role in improving the radiation efficiency of antennas and also allows the control of unwanted side and back lobes in the pattern. This capability of EBG structures can be evaluated experimentally using surface wave measurements.

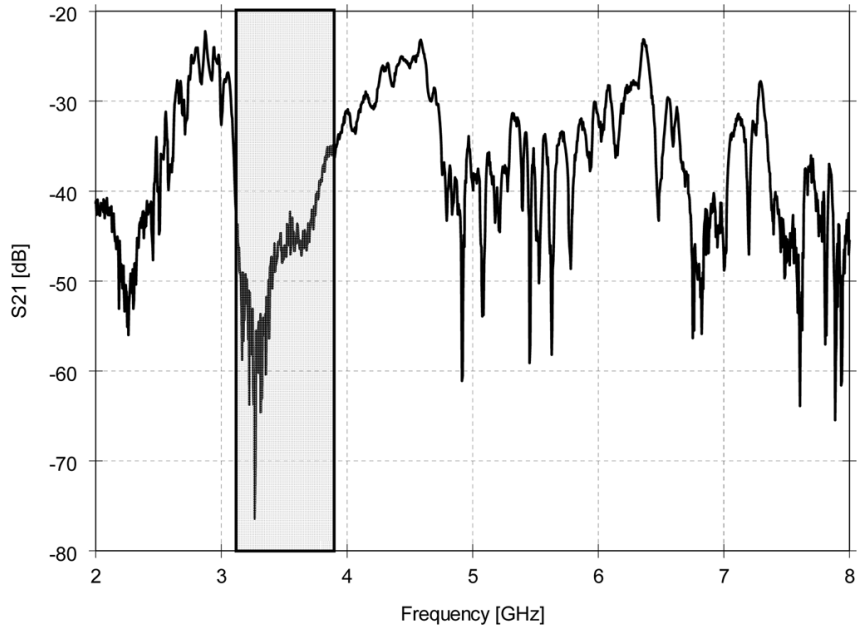

(a)

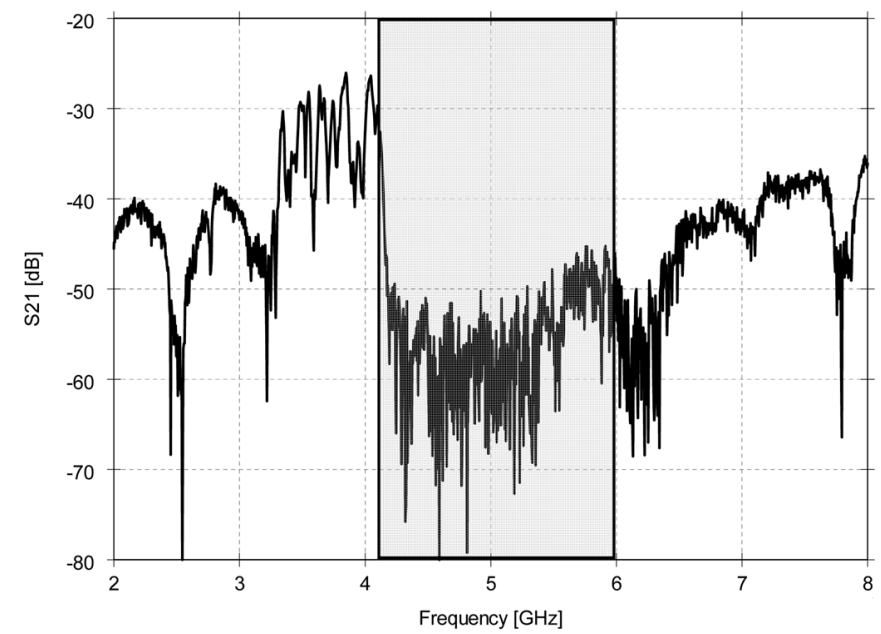

(b)

Fig. 7. TE Surface wave transmission on an EBG surface (a) polar-EBG $\mathrm{k}=$ 0.5 (b) conventional EBG.

To perform these surface wave measurements a $12 \times 12$ array with periodicity $T=7.5 \mathrm{~mm}$ was etched on a Taconic substrate (TLE-95) of thickness $3.18 \mathrm{~mm}$ and relative permittivity $\varepsilon_{r}=2.95$. The EBG property of the fabricated prototype was measured using the coaxial monopole probe methodology from [1]. The measured responses for the polar-EBG and conventional EBG are shown in Fig. 7. The area shaded gray shows the predicted stop-band edges as calculated using (1) and (2). The measured reflection coefficient obtained by means of the coaxial probes shows good agreement with the theoretical stop-band values. Additionally, these results clearly show the presence of a bandgap for the fabricated prototypes.

The conventional EBG shows a bandgap between 4.13 and $6.0 \mathrm{GHz}$ while the polar-EBG with $k=0.5$ demonstrates a bandgap with band edges at $3.06 \mathrm{GHz}$ and $3.8 \mathrm{GHz}$ (Fig. 7). The stop-bands derived from the measurement results agree well with those in the dispersion diagram determined via simulations. The minor discrepancies found can be partly attributed to fabrication tolerances and due to the finite array used in the measurements, as compared to the infinite array simulated. 


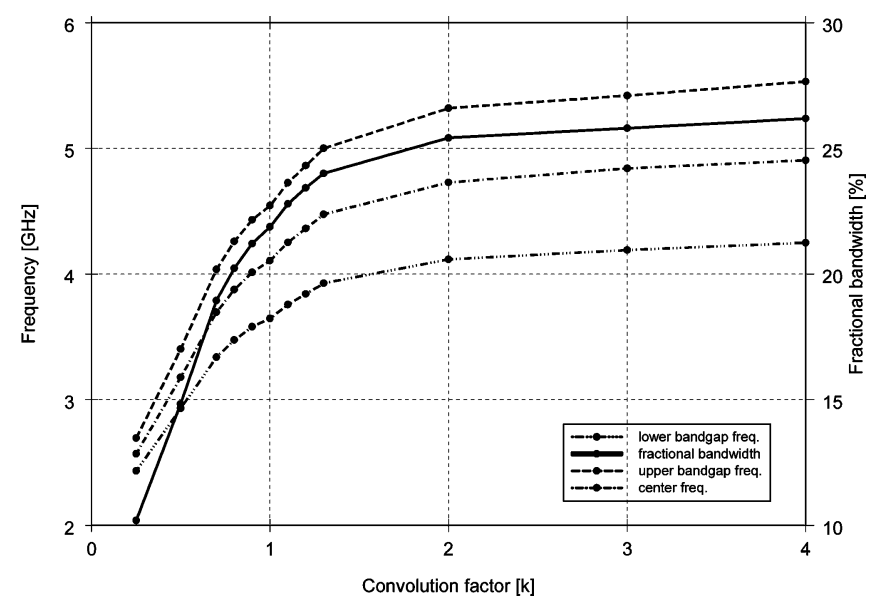

Fig. 8. Effect of convolution factor on the fundamental stop-band of the polarEBG.

\section{BANDGAP TUNING BY VARYING THE CONVOLUTION FACTOR}

A convenient means of controlling the resonant frequency and bandgap of polar-EBG structures is through the use of the convolution factor $k$. This convolution factor offers an additional degree of freedom over the conventional EBG and can be used to design structures for arbitrary frequencies. As discussed in Section II, the convolution factor controls the length of the embedded spiral-slot which contributes proportionally to the capacitance. In this case we consider a polar-EBG on identical substrate to that described in the previous section and again with periodicity $7.5 \mathrm{~mm}$. The spiral-slot width selected was 0.5 $\mathrm{mm}$ and the convolution factor $(k)$ was varied from 0.25 to 4 .

The effect of the convolution factor on the resonant frequency and bandwidth was investigated via simulation using the TLM technique with periodic boundaries at the edges of the unit cell. Note that for $k>4$ the simulation results are almost indistinguishable from the results for the standard square patch element. This can be confirmed by noting the slot length in Fig. 4 is approximately $=1$. Values of $k<0.25$ were not simulated as this would have removed an excessive amount of metal from the array. By considering the reflection phase and measuring the center frequency and bandwidth the bandgaps for different convolution factors were obtained. The results of these simulations are shown plotted in Fig. 8.

It is clearly shown that as $k$ is reduced the resonant frequency decreases in a nonlinear fashion. The greatest rate of change occurs for $k<2$ so this could be considered a lower bound for useful modification. At the lowest values of $\mathrm{k}$ the resonant frequency is still reducing, however this corresponds to increasingly spiraled structures which become difficult to manufacture and the slot width becomes physically unobtainable.

Another important consideration at the left hand side of the plot is the decrease in fractional bandwidth which falls off even more rapidly than the frequency. This reduction in fractional bandwidth is particularly pronounced for $k \leq 1$. Fig. 9 illustrates this effect, demonstrating in addition that when $k=0.25$, a second stop-band appears. This is an indication that the next stop band is also reduced in frequency.

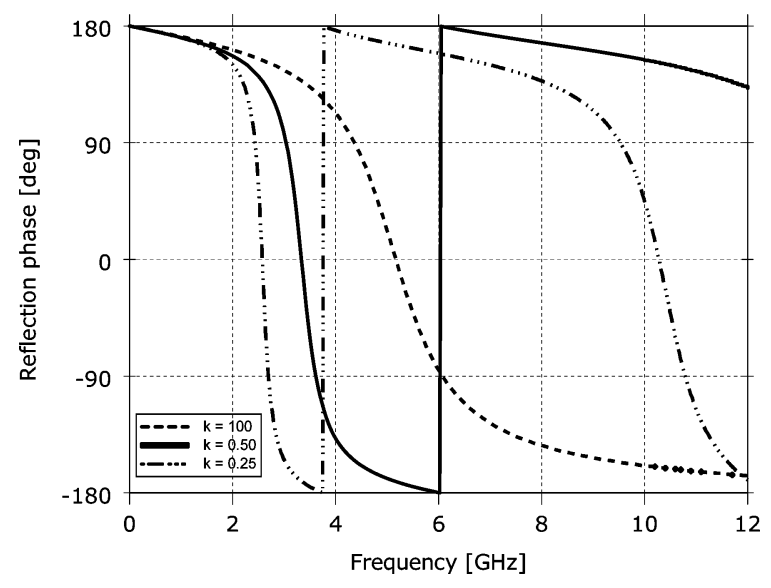

Fig. 9. Reflection phase variation with curve order.

\section{ANGULAR STABILITY}

EBG surfaces do not exhibit uniform surface impedance with respect to different spatial harmonics radiated by antennas [17]. The resonant frequency at which PMC effects are observed depends on the incidence angle, and therefore the resultant interaction of the EBG surface and antenna will be a summation of in-phase and out-of-phase effects [30]. Angular dependence is consequently an important characteristic to be determined for an EBG structure. The angular dependence of the surface impedance can be considered separately for both E-plane (transverse electric, TE) and H-plane (transverse magnetic, TM) polarized waves. For the polar EBG the angular dependence has been determined by applying the methodology in [31]. A frequency domain solver is used to apply incident waves at several incident angles in the range $0-60^{\circ}$ for both TE and TM and the resonant frequency of the EBG is determined with the objective of determining the change in frequency and the total amount of deviation observed for a range of incident angles.

Table II presents the absolute deviation and relative deviation of the resonant frequency for the polar EBG for various $k$ values. The reference case is the $k \rightarrow \infty$ case which corresponds to the standard mushroom EBG. These reference results are in good agreement with those presented by Simovski et al. [31]. In the case of the polar EBG, it is notable that in the case of both TE and TM, the polar spiral pattern has the desirable effect of reducing the angular dependence. The effect is a general trend to reduce the deviation for decreasing $k$. Also, for the case $k=0.25$ the percentage deviation in the resonant frequency for TM and TE is very similar and hence this is a useful design. In comparison to the spiral designs in [30], the polar-EBGs demonstrated greater angular stability for both $k=0.25$, and $k=0.5$ in both TE and TM incident fields. In addition, the polar designs have better angular stability for the case for $k=1.0$. The angular stabilization effect observed in both the TE and TM instances in the polar surface is consistent with the observations in [31] where the excitation of the vias causing, electric currents (TM) and magnetic currents (TE) was produced. 
TABLE I

BANDGAP CHARACTERISTICS ANALYSIS THROUgh REFLECTION PHASE ANALYSIS

\begin{tabular}{|c|c|c|c|c|}
\hline Structure & $\begin{array}{c}\text { Bandgap centre } \\
\text { frequency } f_{c}[\mathrm{GHz}]\end{array}$ & Reduction in $f_{c}$ & Bandgap width $[\mathrm{GHz}]$ & Bandgap as \% of $f_{c}$ \\
\hline Conventional EBG & 5.167 & - & 1.72 & $33.3 \%$ \\
\hline Polar-EBG, $k=0.5$ & 3.62 & $30 \%$ & 0.64 & $17.1 \%$ \\
\hline
\end{tabular}

TABLE II

Relative and Absolute DeViation of Resonant Frequency for DifFerent Values of the Convolution Factor, $k$

\begin{tabular}{|c|c|c|c|c|}
\hline Convolution Factor $[k]$ & \multicolumn{2}{|c|}{ TE-Polarized } & \multicolumn{2}{c|}{ TM-Polarized } \\
\cline { 2 - 5 } & $\begin{array}{c}\text { Absolute deviation } \\
{[\mathrm{GHz}]}\end{array}$ & $\begin{array}{c}\text { Relative deviation } \\
{[\%]}\end{array}$ & $\begin{array}{c}\text { Absolute deviation } \\
{[\mathrm{GHz}]}\end{array}$ & $\begin{array}{c}\text { Relative deviation } \\
{[\%]}\end{array}$ \\
\hline$k \rightarrow \infty$ & 0.217 & 4.00 & 0.073 & 1.44 \\
\hline$k=1.0$ & 0.066 & 1.22 & 0.043 & 1.07 \\
\hline$k=0.5$ & 0.023 & 0.43 & 0.024 & 0.74 \\
\hline$k=0.25$ & 0.004 & 0.07 & 0.002 & 0.08 \\
\hline
\end{tabular}

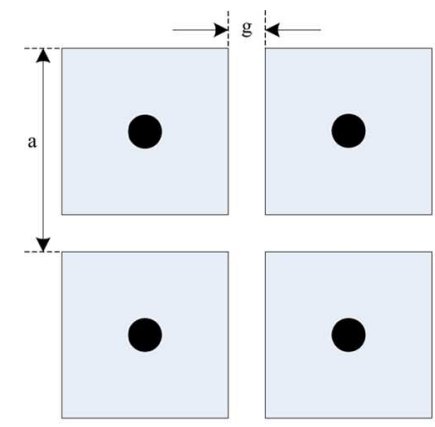

(a)

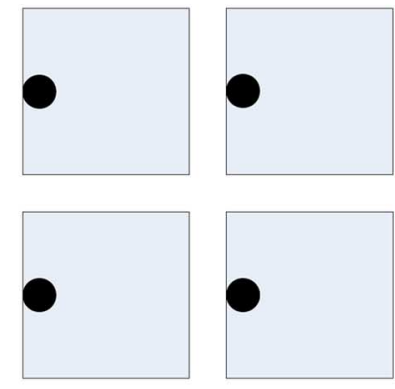

(c)

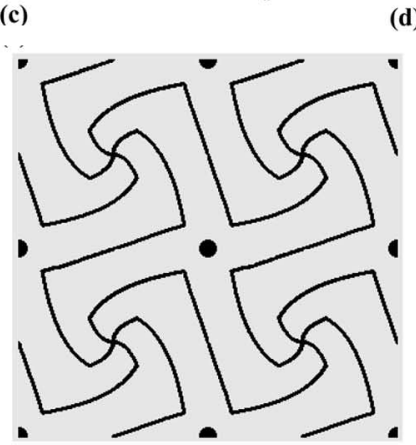

(e)

Fig. 10. Schematics of artificial magnetic conductors (AMC) surfaces: (a) Conventional mushroom structure, (b) spiral-like EBG, (c) edge-located vias, (d) second order Hilbert curve, (e) polar-EBG.

\section{COMPARISON With KNOWN LOW-FrEQUENCY EBG STRUCTURES}

Fig. 10 shows schematic diagrams of five different types of EBG, which were designed on substrates with thickness $t=$
TABLE III

Design SPECIFICATIONS FOR EACH EBG Unit CELl STRUCtURE

\begin{tabular}{l|l}
\hline $\begin{array}{l}\text { EBG Type } \\
\text { Conventional } \\
\text { mushroom }\end{array}$ & $\begin{array}{l}\text { Design parameters } \\
\mathrm{a}=18.07 \mathrm{~mm}, \mathrm{~g}=0.2 \mathrm{~mm}\end{array}$ \\
$\begin{array}{l}\text { Spiral-like } \\
\text { Edge-located vias }\end{array}$ & $\mathrm{a}=4.95 \mathrm{~mm}, \mathrm{~g}=0.18 \mathrm{~mm}$ \\
Polar-EBG & $\mathrm{a}=10.6 \mathrm{~mm}, \mathrm{~g}=0.2 \mathrm{~mm}$ \\
& $\mathrm{a}=8 \mathrm{~mm}, \mathrm{~g}=0.2 \mathrm{~mm}$ \\
Second order Hilbert & $\mathrm{k}=0.5$ \\
curve & $\mathrm{a}=13.65 \mathrm{~mm}, \mathrm{~L}_{\mathrm{h}}=12.65 \mathrm{~mm}$ \\
\hline
\end{tabular}

$2.0 \mathrm{~mm}$ and relative permittivity $\varepsilon_{\mathrm{r}}$ of 2.65 . The radius of the via pins, where present, was kept constant at $0.4 \mathrm{~mm}$. The lattice in Fig. 10(a) is the conventional mushroom structure (as in [3]) which consists of square metal patches connected to the ground plane by metal via pins in the center of the patch. The lattice in Fig. 10(b) is the spiral-like EBG structure proposed by Zheng et al. [33], while the lattice in Fig. 10(c) consists of metal patches with offset metallic vias [34]. Additionally, Fig. 10(d) is the Hilbert curve based high impedance metamaterial surface of iteration order 2 as in [2]. These were compared to the polar-EBG with warped spirals having $k=0.5$.

The EBG structures were all designed to operate at $3.25 \mathrm{GHz}$ and were modeled and optimized using a full-field TLM technique. The final design parameters for each of these surfaces are summarized in Table III. The different geometries were evaluated for plane wave incidence by determining the reflection phase. The bandwidth was calculated from the simulation results by observing the convention of the phase being $\pm 90^{\circ}$ from the phase at the center frequency. The results are summarized in Table IV. The size reduction figures quoted are the percentage of the square unit cell for the geometry measured relative to the conventional mushroom EBG. It is worth noting that the Hilbert curve and edge-located via structures are asymmetrical and therefore necessitate two different orthogonal polarizations making the resonant frequency polarization dependent. In this study, for both the Hilbert curve and edge-located via structures, only the polarization that yields a resonant frequency of $3.25 \mathrm{GHz}$ was considered. The results of the parametric study show that each of the four modified EBG structures has a much lower fractional bandwidth than the conventional mushroom type. The second-order Hilbert curve demonstrates 
TABLE IV

Simulated BANDwidths ANd Cell Size Reduction of EBG Structures

\begin{tabular}{|l|c|c|c|c|}
\hline Structure & $\begin{array}{c}\text { Start } \\
\text { frequency } \\
f_{s}[\mathrm{GHz}]\end{array}$ & $\begin{array}{c}\text { End } \\
\text { Frequency } \\
f_{e}[\mathrm{GHz}]\end{array}$ & $\begin{array}{c}\text { Fractional } \\
\text { Bandwidth }\end{array}$ & $\begin{array}{c}\text { Size } \\
\text { reduction }\end{array}$ \\
\hline $\begin{array}{l}\text { Conventional } \\
\text { EBG }\end{array}$ & 2.87 & 3.61 & $22.7 \%$ & - \\
\hline $\begin{array}{l}\text { Spiral-like } \\
\text { EBG }\end{array}$ & 3.11 & 3.38 & $8.3 \%$ & $72.8 \%$ \\
\hline $\begin{array}{l}\text { Edge located } \\
\text { vias }\end{array}$ & 3.08 & 3.39 & $9.3 \%$ & $41.3 \%$ \\
\hline $\begin{array}{l}\text { Polar-EBG } \\
\text { (k=0.5) }\end{array}$ & 3.11 & 3.40 & $9.0 \%$ & $55.7 \%$ \\
\hline $\begin{array}{l}\text { Hilbert curve } \\
\text { order 2 }\end{array}$ & 3.2 & 3.32 & $3.6 \%$ & $24.4 \%$ \\
\hline
\end{tabular}

the least fractional bandwidth at 3.6\%. The edge-located via and Polar-EBG at $k=0.5$ show remarkably similar fractional bandwidths but with the Polar-EBG having a higher cell size reduction at nearly $56 \%$. The spiral-like EBG exhibits the greatest cell size reduction $\approx 73 \%$ while the Hilbert-curve shows the least cell size reduction at $24 \%$. Although the edge-located via EBG marginally performs better than the polar-EBG structure in terms of bandwidth, the performance of the Polar-EBG is still better than that of the spiral-like EBG and Hilbert curve in terms of the fractional bandwidth.

In the case of the Hilbert-curve, the polar-EBG performs better, because of the greater utilization of the patch area to increase surface capacitance while the Hilbert-curve relies more on its inductive nature. The polar-EBG also has the benefit of being symmetrical and is therefore not polarization dependent while the Hilbert-curve and edge-located via EBGs are polarization dependent. This characteristic may be important to some applications.

The difference in performance of the Spiral-like EBG and polar-EBG can be understood by analyzing each individual structure. The polar-EBG is a direct derivate of the conventional mushroom structure when $k \rightarrow \infty$. The gaps between the patches have been tessellated, and warped in order to increase the capacitance by making better use of the patch area. In the case of the spiral-like EBG, apart from increasing the lateral length of the patch element, interleaving has also been incorporated to a greater degree into the structure to increase the overall capacitance.

\section{CONCLUSION}

In this paper, a novel EBG structure designed using polar curves and mapping functions has been presented and evaluated. Simulations and experiments have been performed which demonstrate that as $k$ is reduced, the angular stability of the surface improves and the resonant frequency reduces, however, as found in other designs, this comes at the cost of reduced bandwidth. In this regard, the polar EBG performs at least as well as the others studied and in some cases better. One of the major advantages in this method of defining the EBG geometry is the ability to adjust the frequency by changing only the slot length via a simple, single parameter, $k$. It is of course possible to adjust the slot width and substrate thickness however the availability of substrate and manufacturing tolerances then become more important when tuning the surface.

\section{ACKNOWLEDGMENT}

The authors gratefully thank Dr. A. Chauraya for help with the prototypes and measurements. They also wish to thank Taconic who kindly donated the laminate samples for the designs and CST for the simulation software.

\section{REFERENCES}

[1] D. F. Sievenpiper, "High-Impedance Electromagnetic Surfaces," Ph.D. dissertation, Univ. California, Los Angeles, 1999.

[2] J. McVay, N. Engheta, and A. Hoorfar, "High impedance metamaterial surfaces using Hilbert-curve inclusions," IEEE Microw. Wireless Compon. Lett., vol. 14, pp. 130-132, 2004.

[3] D. Sievenpiper, L. Zhang, R. F. J. Broas, N. G. Alexopolous, and E. Yablonovitch, "High-impedance electromagnetic surfaces with a forbidden frequency band," IEEE Trans. Microw. Theory Tech., vol. 47, pp. 2059-2074, 1999.

[4] S. Wang, A. P. Feresidis, J. C. Vardaxoglou, and G. Goussetis, "Artificial magnetic conductors for low-profile resonant cavity antennas," in AP-S Int. Symp. (Digest) IEEE Antennas Propag. Society, 2004, vol. 2, pp. 1423-1426.

[5] F. Yang and Y. Rahmat-Samii, "Reflection phase characterization of an electromagnetic band-gap (EBG) surface," in Proc. IEEE Int. Symp. Antennas Propag., 2002, pp. 744-747.

[6] L. Yang, M. Fan, F. Chen, J. She, and Z. Feng, "A novel compact electromagnetic-bandgap (EBG) structure and its applications for microwave circuits," IEEE Trans. Microw. Theory Tech., vol. 53, pp. 183-190, 2005.

[7] Y. Yao, X. Wang, and Z. Feng, "A novel dual-band compact electromagnetic bandgap (EBG) structure and its application in multi-antennas," in Proc. IEEE Int. Symp. Antennas and Propag. Society, 2006, pp. $1943-1946$.

[8] L. Inclan-Sanchez, E. Rajo-Iglesias, J. L. Vazquez-Roy, and V. Gonzalez-Posadas, "Design of periodic metallo-dielectric structure for broadband multilayer patch antenna," Microw. Opt. Technol. Lett., vol. 44, pp. 418-421, 2005.

[9] R. F. J. Broas, D. F. Sievenpiper, and E. Yablonovitch, "A high-impedance ground plane applied to a cellphone handset geometry," IEEE Trans. Microw. Theory Tech., vol. 49, pp. 1262-1265, 2001.

[10] J. R. Sohn, H. Tae, J. Lee, and J. Lee, "Comparative analysis of four types of high-impedance surfaces for low profile antenna applications," in AP-S IEEE Int. Symp. (Digest) Antennas Propag. Society, 2005, vol. 1A, pp. 758-761.

[11] W. Zhang, J. Mao, and X. Sun, "Patch antenna array embedded on a high-impedance ground plane," J. Electromagn. Waves Applicat., vol. 19, pp. 2007-2014, 2005.

[12] F. Yang and Y. Rahmat-Samii, "Microstrip antennas integrated with electromagnetic band-gap (EBG) structures: A low mutual coupling design for array applications," IEEE Trans. Antennas Propag., vol. 51, pp. 2936-2946, 2003.

[13] R. Gonzalo, P. De Maagt, and M. Sorolla, "Enhanced patch-antenna performance by suppressing surface waves using photonic-bandgap substrates," IEEE Trans. Microw. Theory Tech., vol. 47, pp. 2131-2138, 1999.

[14] F. Yang and Y. Rahmat-Samii, "Microstrip antennas integrated with electromagnetic band-gap (EBG) structures: A low mutual coupling design for array applications," IEEE Trans. Antennas Propag., vol. 51, pp. 2936-2946, 2003.

[15] F. Yang, K. Ma, Y. Qian, and T. Itoh, "A novel TEM waveguide using uniplanar compact photonic-bandgap (UC-PBG) structure," IEEE Trans. Microw. Theory Tech., vol. 47, pp. 2092-2098, 1999.

[16] F. Yang, K. Ma, Y. Qian, and T. Itoh, "A uniplanar compact photonic-bandgap (UC-PBG) structure and its applications for microwave circuit," IEEE Trans. Microw. Theory Tech., vol. 47, pp. 1509-1514, 1999.

[17] G. K. Palikaras, A. P. Feresidis, and J. C. Vardaxoglou, "Cylindrical electromagnetic bandgap structures for directive base station antennas," IEEE Antennas Wireless Propag. Lett., vol. 3, pp. 87-89, 2004.

[18] T. Kamgaing and O. M. Ramahi, "Design and modeling of high-impedance electromagnetic surfaces for switching noise suppression in power planes," IEEE Trans. Electromagn. Compat., vol. 47, pp. 479-489, 2005. 
[19] T. Kamgaing and O. M. Ramahi, "A novel power plane with integrated simultaneous switching noise mitigation capability using high impedance surface," IEEE Microw. Wireless Compon. Lett., vol. 13, pp. 21-23, 2003.

[20] R. Abhari and G. V. Eleftheriades, "Metallo-dielectric electromagnetic bandgap structures for suppression and isolation of the parallel-plate noise in high-speed circuits," IEEE Trans. Microw. Theory Tech., vol. 51, pp. 1629-1639, 2003.

[21] D. Sievenpiper, "Review of theory, fabrication, and applications of high-impedance ground planes," in Metamaterials, Physics and Engineering Explorations, N. Engheta and R. W. Ziolkowski, Eds. : IEEE Press, 2006, pp. 295-297.

[22] M. F. Abedin, M. Z. Azad, and M. Ali, "Wideband smaller unit-cell planar EBG structures and their application," IEEE Trans. Antennas Propag., vol. 56, pp. 903-908, 2008.

[23] D. J. Kern, D. H. Werner, M. J. Wilhelm, L. Lanuzza, and A. Monorchio, "The design synthesis of multiband artificial magnetic conductors using high impedance frequency selective surfaces," IEEE Trans. Antennas Propag., vol. 53, pp. 8-17, 2005.

[24] S. Tse, Y. Hao, and C. Parini, "Mushroom-like high-impedance surface (HIS) with slanted vias," in Proc. LAPC Antennas and Propag. Conf., Loughborough, 2007, pp. 309-312.

[25] D. J. Kern, D. H. Werner, and M. J. Wilhelm, "Active negative impedance loaded EBG structures for the realization of ultra-wideband artificial magnetic conductors," in IEEE AP-S Int. Symp. (Digest) Antennas Propag. Society, 2003, vol. 2, pp. 427-430.

[26] G. S. A. Shaker and S. Safavi-Naeini, "Reduced size electromagnetic bandgap (EBG) structures for antenna applications," in Proc. Canadian Conf. Elect. and Comput. Eng., 2005, pp. 1198-1201.

[27] D. J. Kern, D. H. Werner, K. H. Church, and M. J. Wilhelm, "Genetically engineered multiband high-impedance frequency selective surfaces," Microw. Opt. Technol. Lett., vol. 38, pp. 400-403, 2003.

[28] J. McVay, A. Hoorfar, and N. Engheta, "Radiation characteristics of microstrip dipole antennas over a high-impedance metamaterial surface made of Hilbert inclusions," in IEEE MTT S Int. Microw. Symp. Dig., 2003, vol. 1, pp. 587-590.

[29] P. B. Johns, "A symmetrical condensed node for the TLM method," IEEE Trans. Microw. Theory Tech., vol. 35, pp. 370-377, 1987.

[30] M. Hosseini, A. Pirhadi, and M. Hakkak, "Design of an AMC with little sensitivity to angle of incidence using an optimized Jerusalem cross FSS," in IEEE Int. Workshop on Antenna Technology: Small Antennas and Novel Metamaterials, 2006, pp. 245-248.

[31] C. R. Simovski, P. De Maagt, A. A. Sochava, M. Paquay, and S. A. Tretyakov, "Angular stabilisation of resonant frequency of artificial magnetic conductors for TE-incidence," Electron. Lett., vol. 40, pp. 92-93, 2004.
[32] C. R. Simovski, P. De Maagt, and I. V. Melchakova, "High-impedance surfaces having stable resonance with respect to polarization and incidence angle," IEEE Trans. Antennas Propag., vol. 53, pp. 908-914, 2005.

[33] Q.-R. Zheng, Y.-Q. Fu, and N.-C. Yuan, "A novel compact spiral electromagnetic band-gap (EBG) structure," IEEE Trans. Antennas Propag., vol. 56, pp. 1656-1660, 2008.

[34] E. Rajo-Iglesias, L. Inclan-Sanchez, J. Vazquez-Roy, and E. GarciaMuoz, "Size reduction of mushroom-type EBG surfaces by using edgelocated vias," IEEE Microw. Wireless Components. Lett., vol. 17, pp. 670-672, 2007

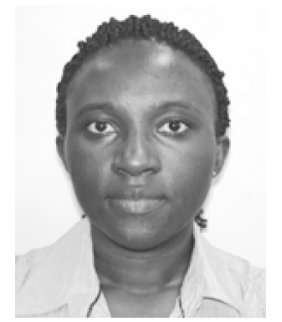

Charity B. Mulenga received the B.Sc. degree in electrical engineering from Makerere University, Uganda, in 2001 and the M.Sc. degree in digital communication systems from Loughborough University, Loughborough, U.K., in 2004, where, since October 2006, she has been working toward the Ph.D. degree.

Her research interests focus on the design of broadband antennas, periodic structures, computational electromagnetics and frequency selective surfaces.

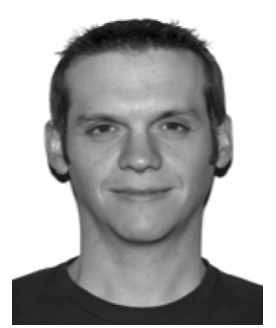

James A. Flint (M'96-SM'07) was born in Holbrook, Derbyshire, U.K., in 1973. He received the M.Eng. and Ph.D. degrees in electronic and electrical engineering from Loughborough University, Loughborough, U.K., in 1996 and 2000, respectively.

He worked in the automotive industry as a Project Engineer and later returned to Loughborough when he was appointed Lecturer in Wireless Systems Engineering following in 2001. He was promoted to Senior Lecturer in 2006 and is currently the Head of the Communications Research Division, Department of Electrical and Electronic Engineering. His research interests span fault-tolerant signal processing, novel acoustic and electromagnetic transducers, metamaterials and electromagnetic compatibility.

Dr. Flint is a Chartered Engineer in the U.K., a member of the Institution of Engineering and Technology, and has acted as a consultant to numerous highprofile companies and government agencies. 